\title{
Robotics and minimally invasive esophageal surgery
}

\author{
Ealaf Shemmeri, Jon O. Wee \\ Department of Thoracic Surgery, Brigham and Women's Hospital, Boston, MA 02115, USA \\ Contributions: (I) Conception and design: Both authors; (II) Administrative support: JO Wee; (III) Provision of study materials or patients: JO Wee; (IV) \\ Collection and assembly of data: E Shemmeri; (V) Data analysis and interpretation: All authors; (VI) Manuscript writing: Both authors; (VII) Final \\ approval of manuscript: Both authors. \\ Correspondence to: Jon O. Wee. Department of Thoracic Surgery, Brigham and Women's Hospital, 75 Francis Street, Boston, MA 02115, USA. \\ Email: jwee@bwh.harvard.edu.
}

\begin{abstract}
The robotic platform has permeated esophageal surgery both in the abdominal and thoracic approaches. The most widely studied entities include achalasia, gastroesophageal reflux disease, hiatal hernia and esophageal cancer. A literature review of robotic surgeries for the management of the above mentioned disorders is presented. Data is limited to meta-analyses, case series, or small prospective trials in the different indications. One exception is a randomized controlled trial looking at outcomes in esophageal cancer being managed with a hybrid robotic versus open approach. Overall differences when comparing laparoscopic or thoracoscopic surgery to robotic are few. These differences are best highlighted in the achalasia and esophageal cancer literature. There are less intraoperative mucosal injuries in robotic Heller myotomy. A large meta analysis found a rate of $1 \%$ versus $24.5 \%$ mucosal injury rate favoring the robotic versus laparoscopic Heller myotomy methods. With respect to esophagectomy data, there is slightly less vocal cord paralysis in the robotic versus MIE data, with a $\mathrm{P}$ value of 0.044 . However, length of stay, intraoperative bleeding and major morbidity are similar across the various indications. Robotic esophageal surgery is a safe alternative to laparoscopic/thoracoscopic techniques. Further large-scale randomized trials are needed to fully ascertain if it yields superior outcomes.
\end{abstract}

Keywords: Foregut; robotic; esophageal surgery

Submitted May 21, 2020. Accepted for publication Jul 10, 2020.

doi: $10.21037 / \mathrm{atm}-20-4138$

View this article at: http://dx.doi.org/10.21037/atm-20-4138

\section{Introduction}

Access to the esophagus has historically been difficult and treacherous. We have seen several major breakthroughs in the field of surgery that have made diseases of the esophagus better evaluated and with less surgical morbidity. The most recent addition to the thoracic surgeon's armamentarium is the robotic platform. Since 2000, it permeated the various foregut indications; achalasia, diverticulum, hiatal hernia, and esophageal cancer $(1,2)$. The experiences into each indication is summarized here, along with unique pitfalls and benefits in contemporary literature.

\section{Benign foregut}

\section{Achalasia}

Achalasia remains a rare neurodegenerative disorder of the lower esophageal sphincter (LES), leading to failure of the esophagus to empty into the stomach. Our understanding of this disorder has dramatically improved with the advent of high-resolution manometry and timed barium swallows. Yet, we still have no plausible etiology or procedure to regain the function of the LES back to baseline. Our surgical procedures at this time remain palliative in 
nature and include endoscopic and surgical techniques. With the introduction of per oral endoscopic myotomy (POEM), we have seen a rise in this novel method for palliation of achalasia. Yet there is concern of higher rates of reflux compared to the traditional surgical approach. The modified Heller myotomy with partial fundoplication has been transformed from the open laparotomy to the laparoscopic version. And now, we have seen the robotic version of this surgery in the literature. Publications are mostly retrospective in nature, with historical comparisons to laparoscopic cohorts for analysis.

Horgan et al. presented a retrospective review, comparing the laparoscopic to robotic achalasia myotomies from 1995 to 2004 (3). Achalasia was diagnosed using patient questionnaires, barium swallow, endoscopy and manometry. Robotic cases were performed in lithotomy position with $38 \mathrm{~mm}$ ports placed in a linear fashion between the umbilicus and xiphoid process. A small incision is made for placement of Nathanson liver retractor in the subxiphoid area. An assistant port is at the left lateral abdomen. Myotomy was done $6 \mathrm{~cm}$ proximally and $2 \mathrm{~cm}$ distally and a Dor fundoplication was fashioned to cover the myotomy. Their laparoscopic port sites were placed in similar fashion. Of the 61 patients who had laparoscopic repair, 10 had intraoperative esophageal mucosal perforation; 9 of which were repaired at the index operation. One patient had conversion to open for the repair. One patient had pneumonia on postoperative day 2. The robotic cohort included 59 patients. There were no intraoperative mucosal injuries, but one patient developed incarcerated incisional hernia on postoperative day 4 and another patient developed transverse colon perforation requiring partial colectomy. Mean hospital stay was 2.2 days for the laparoscopic and 1.5 days for the robotic group. There were no deaths in either group. The laparoscopic group had LES mean preoperative pressure $26 \pm 6 \mathrm{mmHg}$ with postoperative decrease of $10 \pm 1.5, \mathrm{P}$ value $=0.01$. The robotic group had LES mean preoperative pressure was $33 \pm 13 \mathrm{mmHg}$, with a delta decrease of $7.1 \pm 3.8$ with $\mathrm{P}$ value of 0.01 . Each group had 10 patients who complained of gastroesophageal reflux disease (GERD) symptoms, all managed with antisecretory agents. This study was not matched to either patient characteristics or institution (laparoscopic group in Buenos Aires, and robotic group in Chicago), but highlighted objective and subjective results of the two treatment modalities.

A more robust review in the form of a meta-analysis was recently published by Milone et al. over a 24-year span of the robotic achalasia literature (4). They focused on comparative data looking at robotic versus laparoscopic myotomy in achalasia. A pool of 2,625 patients, comprised of 2 prospective trials and 4 retrospective trials were analyzed. Data including patient demographics, intraoperative complications and postoperative outcome measures were recorded. With regards to esophageal mucosal injury, at total of 2/189 (1\%) robotic versus $42 / 171$ (24.5\%) laparoscopic procedures were reviewed, with less injuries in the robotic group with $\mathrm{OR}=0.13, \mathrm{P}<0.001$. Safety of the operations was similar, when looking at intraoperative bleeding and conversion to open surgery. Postoperative recurrence of dysphagia was reported in 18/165 (11\%) robotic and 18/134 (13\%) laparoscopic, without significant differences captured between the two groups; OR $=0.59$, $\mathrm{P}=0.45$. Though the intraoperative mucosal injuries were more frequent in the laparoscopic group, there was no major consequence to the patients as they were repaired during the index procedure and did not alter their recovery. Surgical management of achalasia, short of randomized prospective data, fairs similarly to laparoscopic myotomy, with possibly less likelihood of intraoperative mucosal injuries.

\section{Diverticulum}

Esophageal diverticulum is a rare, benign entity that is often segregated into anatomic presence; proximal and epiphrenic. Epiphrenic diverticula are thought to arise from pulsion physiology and are a false diverticula near the gastroesophageal (GE) junction. Even less common is middle esophageal diverticula that develop due to traction from mediastinal disease. For epiphrenic outpouchings, though the exact etiology is not always known, patients present with concomitant hypercontractile esophagus, achalasia, or hypertensive LES.

A meta-analysis by Chan and colleagues reviewed the non-robotic literature on the surgical management of nonZenker's diverticula (5). In review of 511 patients, they reported diverticulectomy in $89 \%$ of patients, myotomy in $85 \%$, and fundoplication in $69 \%$ of patients (Dor $\mathrm{n}=148$, Belsey Mark IV $\mathrm{n}=100$, Toupet $\mathrm{n}=63$, and Nissen $\mathrm{n}=44)$. Staple line leak occurred in 51/392 (13\%) patients; 16 of which were made NPO and given TPN, 17 had percutaneous drainage, 15 had operative revision, and 3 had endoscopic stenting. There were 14/51 (27.4\%) deaths in the leak group. This was the largest compilation of data on this rare entity, highlighting the variability in its management. Surgical management remains controversial, 
including the proper access (abdominal versus thoracic) and whether a diverticulectomy should be combined with myotomy and fundoplication (6,7). This analysis and others continue to add to the body of literature on the variability of diverticulum management.

Robotic diverticulectomy is limited to case series and case reports. The port set up has been reported as thoracoscopic and laparoscopic. An example of the abdominal approach was reported by Pernazza and colleagues (8). Their robotic case was of a patient with dysphagia, esophageal dysmotility and epiphrenic diverticulum. They placed the patient in modified lithotomy position with a linear placement of robotic ports across the mid-abdomen. An assist port was in the left lateral abdomen. They performed a diverticulectomy using a linear stapler, a distal myotomy, and a Dor fundoplication. They reported no complications from this case.

Thoracic robotic approaches have also been reported. Balci et al. reviewed three cases of esophageal diverticulum thought to arise at mid-portion due to traction physiology from underlying pulmonary disease (9). Patients were placed in left lateral decubitus position and ventilated with a double lumen endotracheal tube. A $12 \mathrm{~mm}$ robotic camera port was placed at the 4th intercostal space at the anterior axillary line. Two $5 \mathrm{~mm}$ robotic working ports were placed at the posterior axillary line at the 5 th and the 8th intercostal spaces. An inferior assist port was placed right above the diaphragm. A linear stapler was used to perform the diverticulectomy. No myotomy or fundoplication was performed in any of the cases as the authors reported no dysphagia or esophageal dysmotility in preoperative evaluations. Pathologic review showed that all three diverticula were true with all layers of the esophagus. One patient had staple line leak resulting in death, and one patient developed pneumonia requiring a 17-day hospital stay.

Cerfolio and colleagues reported their robotic experience on mediastinal pathology, including five patients who underwent diverticulectomy for epiphrenic diverticula (10). Thoracic port placement was done through the right chest in left lateral decubitus position. The ports were at the midaxillary line using all four robotic arms. They performed a diverticulectomy and myotomy. One patient had a $7 \mathrm{~cm}$ diverticulum that was incompletely transected with the linear stapler, requiring conversion to thoracotomy to complete the resection. There was one patient who developed a delayed staple line leak 1 week after discharge, managed with endoscopic stenting and left thoracoscopic decortication. With the robot docked at the patient's back and having the ports face the hiatus, the authors report that the robotic platform provided better visualization of the distal esophagus when compared to VATS.

The robotic platform certainly adds greater dexterity and visualization both in the abdomen and chest views of the esophagus. More studies need to report on what access is optimal; thoracic versus abdominal. The addition of myotomy and fundoplication also has yet to be analyzed in the robotic diverticulectomy literature.

\section{Hiatal hernia and GERD}

The gold standard approach to the surgical management of typical GERD symptoms is laparoscopic Nissen fundoplication. It has been established as a relatively safe and durable procedure. Dysphagia is a problematic complication that may require further treatment such as dilation or revision of the fundoplication.

There are numerous publications on the robotic platform for GERD management, with hiatal hernia repair data being limited to single center retrospective case series $(11,12)$. A meta-analysis reviewed five randomized controlled trials that included 160 patients looking at robotic and laparoscopic surgery for GERD (13). Analysis was conducted on six prospective comparative trials in the literature comparing laparoscopic Nissen fundoplication to robotic. The primary outcome was requirement for re-operation, postoperative mortality, and postoperative dysphagia. Secondary outcomes included operative time, hospital stay, cost, and operative complications within the first 30 postoperative days. There were no mortalities in the analyzed studies. Reoperation rates were similar in laparoscopic and robotic data. Postoperative dysphagia was recorded in three trials, without significant difference between the two surgical modalities. With regards to secondary outcomes, there was no difference in hospital length of stay, or operative complications. Operating time was significantly less in the laparoscopic group with $P$ value $=0.0002$. As expected, cost was higher in the robotic group. The results of this paper shows that robotic Nissen fundoplication is a safe and effective alternative to laparoscopic surgery, but still requires a higher institutional cost to implement.

\section{Esophageal cancer}

Esophageal cancer continues to increase in incidence in the 


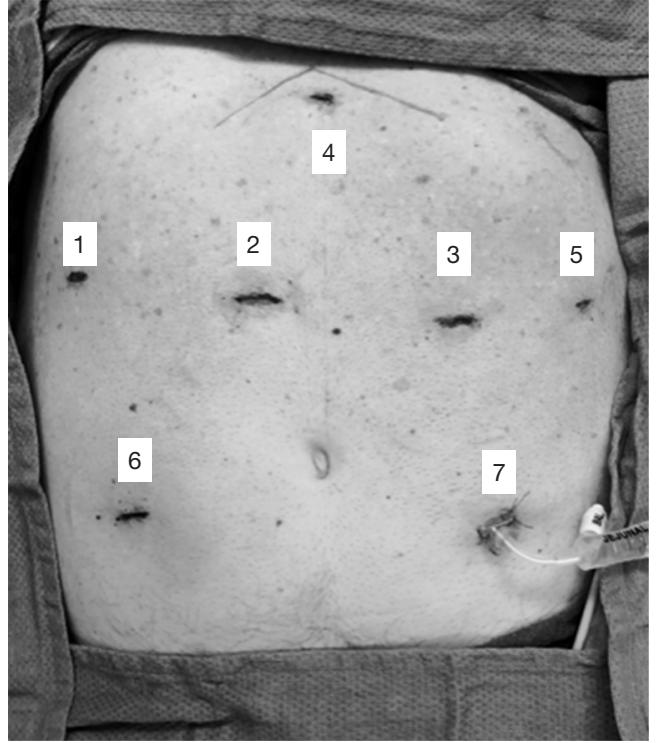

Figure 1 Laparoscopic supine port placement. 1. Right lateral $5 \mathrm{~mm}$ port; $2.12 \mathrm{~mm}$ right paramedian working port; $3.11 \mathrm{~mm}$ camera port; 4. $5 \mathrm{~mm}$ subxiphoid Nathanson liver retractor port; 5. left lateral $5 \mathrm{~mm}$ assist port; $6.12 \mathrm{~mm}$ right lower quadrant endostich port; 7. Jejunostomy tube.

United States. Adenocarcinoma predominates the histology, linked to obesity and long-standing Barrett's esophagus near the GE junction. Esophagectomy remains a critical element of multimodality treatment. The transthoracic Ivor Lewis approach is the most common technique. Reports on approaches range from open laparotomy/thoracotomy to exclusively minimally invasive using laparoscopy/ thoracoscopy and all iterations in between.

In 2012, Luketich and colleagues reviewed over a thousand cases of minimally invasive esophagectomies done either as transthoracic Ivor Lewis or modified McKeown from 1996 to 2011 at the University of Pittsburgh Medical Center (14). McKeown minimally invasive esophagectomy (MIE) was performed in 481/1,011 patients, while Ivor Lewis MIE was done in 530/1,011 patients. Conversion to open was reported in 45/1,011 (4.5\%) of cases in either the chest or abdomen and were similar rates in between the two surgical groups. There were no intraoperative mortalities, but there was a 30 -day mortality of $1.68 \%$, which was lower in the Ivor Lewis MIE group at $0.9 \%$. This did not reach statistical significance. An R0 resection was achieved in $98 \%$ of the series, with median 21 lymph nodes resected. Rates of major complications such as myocardial infarction, need for splenectomy to control bleeding, acute respiratory distress syndrome (ARDS), and empyema were similar between McKeown and Ivor Lewis MIE. Gastric necrosis occurred in 15/481 (3\%) of the McKeown group and 9/530 (2\%) of the Ivor Lewis group without statistical significance. The only complication that was significantly different between the two groups was vocal cord paralysis, occurring more frequently in the McKeown MIE group in 37/481 (8\%) patients, compared to $5 / 530(1 \%)$ of the Ivor Lewis patients with $\mathrm{P}$ value $\leq 0.001$. The median follow up was 20 months with overall survival at 1 year was $86 \%$ for stage $0,89 \%$ for stage I, $80 \%$ for stage IIa, $76 \%$ for stage IIb, $63 \%$ for stage III, and $44 \%$ for stage IV.

Their data is used as one of the benchmarks in minimally invasive esophagectomy data to compare robotic experience (15). Several papers report on large retrospective case series with the use of the robotic platform in esophagectomy (16).

Okusanya et al. reported on 23 patients who had Ivor Lewis esophagectomy done robotically for both the abdominal and thoracic portion (17). Their anastomosis was performed using a circular end to end stapler. They reported no 30-day mortality, and one patient developed a leak. Once the learning curve for the robotic platform is achieved, there is a clear reduction of morbidity when compared to open esophagectomy.

We analyzed our early experience on transthoracic esophagectomy, using laparoscopic and robot-assist thoracoscopic techniques in 20 patients (see Figures 1 and 2) (18). There was no operative mortality and our most common complications were atrial fibrillation at $15 \%$ of cases and $10 \%$ for pneumonia. There were no anastomotic leaks. In addition to the standardized use of a circular end-end stapled anastomosis, we placed an omental flap sandwiched between the anastomosis and the airway. We feel this added step greatly reduces development of anastomotic leak.

A recent meta-analysis by Jin and colleagues examined the literature, focusing on operative time, blood loss, length of stay, number of harvested lymph nodes, 30-day postoperative mortality, and achievement of R0 resection (19). A total of 8 out of 906 studies were included in this analysis that reported comparative data between robotic and MIE technique. Length of stay, operative time, and mortality were not statistically different between the two modalities. Number of lymph nodes harvested was also similar. Blood loss was lower in the robotic group with $\mathrm{P}=0.0075$. Vocal cord paralysis was more frequent in the MIE group with $\mathrm{P}=0.044$. However, other complications 


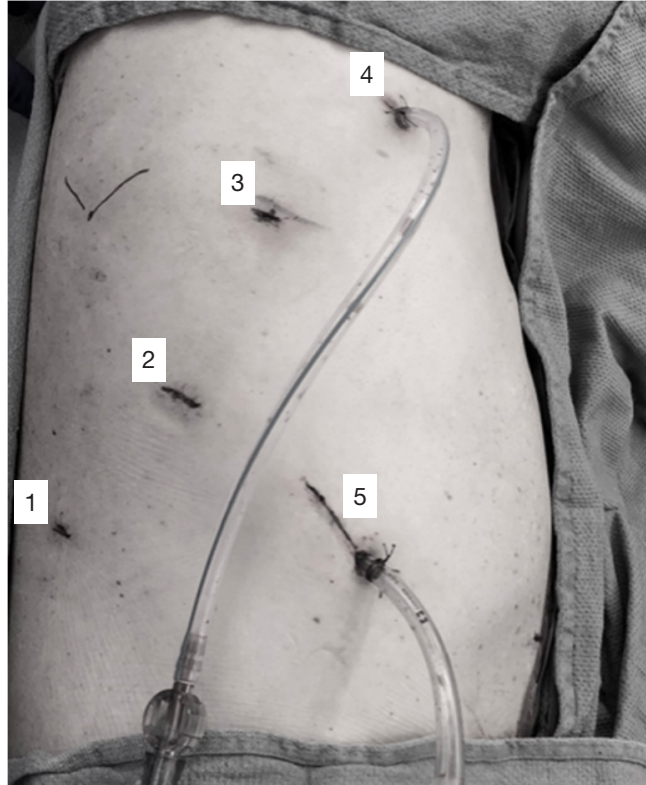

Figure 2 Robotic left lateral decubitus thoracic port placement. 1. Tip-up forceps $8 \mathrm{~mm}$ port; 2. Cadiere forceps $12 \mathrm{~mm}$ port; 3. zerodegree camera $8 \mathrm{~mm}$ port; 4 . fenestrated bipolar $8 \mathrm{~mm}$ port; 5 . assistant/extraction port.

including pneumonia, arrhythmia, chylothorax, empyema, and anastomotic leak were not statistically different between MIE and robotic esophagectomy. Greater visualization is suggested to be better, offering a better view for lymph node dissection without traction injury to the recurrent laryngeal nerve.

The only randomized prospective trial for esophagectomy is reported by van der Sluis (The ROBOT trial) (20). This is a prospective analysis of 56 patients who underwent robotic-assisted esophagectomy versus 56 matched patients who underwent traditional open esophagectomy. The robotic arm was performed using robotic mobilization of the thoracic esophagus followed by laparoscopic and open neck resection and hand-sewn neck anastomosis. The primary endpoint was occurrence of grade II overall surgery-related complications. Almost $60 \%$ of the robot group developed complications compared to $80 \%$ of the open group with a $\mathrm{P}$ value of 0.02 . Their most common complications were pulmonary, accounting for $32 \%$ (pneumonia, pulmonary embolism) of patients in the robotic group and 58\% (pneumonia, pneumothorax, pulmonary embolism, ARDS) of the open group, $\mathrm{P}$ value $=0.005$. Interestingly, anastomotic leak occurred in about
$20 \%$ of cases in each arm. Quality of life questionnaires, however, favored the robot arm. Overall and disease-free survival was similar in both groups. The authors concluded that their robotic technique greatly reduced the morbidity of esophagectomy when compared to traditional open esophagectomy.

Long term complications reported in the literature on MIE include development of hiatal hernia. This is thought to occur more frequently than in open esophagectomy due to the lack of tactile ability to reduce the redundancy of the gastric conduit after the creation of the anastomosis. Occurrence of hiatal hernia following esophagectomy was reported by Oor and colleagues in a meta-analysis (21). They reviewed a literature search on the occurrence of hiatal hernia after open or robotic esophagectomy. The primary outcome was the difference in incidence of hiatal hernia in open versus robotic esophagectomy. Open surgeries included transhiatal, McKeown, transthoracic, Ivor Lewis. They analyzed a total of 26 studies, one randomized controlled trial, one prospective trial and 24 retrospective cohort studies between 1985 to 2015 . A total of 6,058 patients were included, with 240 developed hiatal hernia. The incidence of hernia in the open group ranged from $0-12 \%$ with median time to development was 21 months. Among the MIE 20 studies, the rate of hiatal hernia ranged from $0-26 \%$ at median follow up time of 8.8 months. Their minimally invasive esophagectomy data, only had one report on robotic MIE that included 36 patients who underwent robotic transhiatal esophagectomy. A total of 7/36 (19\%) patients developed hiatal hernia that was repaired laparoscopically with cruroplasty and mesh placement. From this limited data, it appears that rates of hiatal hernia following robotic MIE is similar to that of MIE.

\section{Conclusion}

Robotic surgery has proven to be a key addition to the management of esophageal diseases. Though there are improvements in quality of life measures, it has yet to outperform the laparoscopic/thoracoscopic technique. Further matched comparative studies are needed to fully evaluate this question.

\section{Acknowledgments}

Funding: None. 


\section{Footnote}

Provenance and Peer Review: This article was commissioned by the Guest Editor (Dr. Ory Wiesel) for the series "Innovations and Updates in Esophageal Surgery" published in Annals of Translational Medicine. The article was sent for external peer review organized by the Guest Editor and the editorial office.

Conflicts of Interest: Both authors have completed the ICMJE uniform disclosure form (available at http://dx.doi. org/10.21037/atm-20-4138). The series "Innovations and Updates in Esophageal Surgery" was commissioned by the editorial office without any funding or sponsorship. The authors have no other conflicts of interest to declare.

Ethical Statement: The authors are accountable for all aspects of the work in ensuring that questions related to the accuracy or integrity of any part of the work are appropriately investigated and resolved.

Open Access Statement: This is an Open Access article distributed in accordance with the Creative Commons Attribution-NonCommercial-NoDerivs 4.0 International License (CC BY-NC-ND 4.0), which permits the noncommercial replication and distribution of the article with the strict proviso that no changes or edits are made and the original work is properly cited (including links to both the formal publication through the relevant DOI and the license). See: https://creativecommons.org/licenses/by-nc-nd/4.0/.

\section{References}

1. Rebecchi F, Allaix ME, Morino M. Robotic technological aids in esophageal surgery. J Vis Surg 2017;3:7.

2. Cadiere GB, Himpens J, Vertruyen M, et al. Evaluation of telesurgical (robotic) Nissen fundoplication. Surg Endosc 2001;15:918-23.

3. Horgan S, Galvani C, Gorodner MV, et al. Roboticassisted Heller myotomy versus laparoscopic Heller myotomy for the treatment of esophageal achalasia: A multicenter study. J Gastrointest Surg 2005;9:1020-9; discussion 1029-30.

4. Milone M, Manigrasso M, Vertaldi S, et al. Robotic versus laparoscopic approach to treat symptomatic achalasia: systematic review with meta-analysis. Dis Esophagus 2019;32:1-8.

5. Chan DS, Foliaki A, Lewis WG, et al. Systematic review and meta-analysis of surgical treatment of nonZenker's oesophageal diverticula. J Gastrointest Surg 2017;21:1067-75.

6. Achim V, Aye RW, Farivar AS, et al. A combined thoracoscopic and laparoscopic approach for high epiphrenic diverticula and the importance of complete myotomy. Surg Endosc 2017;31:788-94.

7. Fernando HC, Luketich JD, Samphire J, et al. Minimally invasive operation for esophageal diverticula. Ann Thorac Surg 2005;80:2076-80.

8. Pernazza G, Monsellato I, Pende V, et al. Fully robotic treatment of an epiphrenic diverticulum; report of a case. Minim Invasive Ther Allied Technol 2012;21:96-100.

9. Balci B, Kilinc G, Calik B, et al. Robotic-assisted transthoracic esophageal diverticulectomy. JSLS 2018;22:e2018.00002.

10. Cerfolio RJ, Bryant AS, and Minnich DJ. Operative techniques in robotic thoracic surgery for inferior or posterior mediastinal pathology. J Thorac Cardiovasc Surg 2012;143:1138-43.

11. Morino M, Pellegrino L, Giaccone C, et al. Randomized clinical trial of robot-assisted versus laparoscopic Nissen fundoplication. Br J Surg 2006;93:553-8.

12. Frazzoni M, Conigliaro R, Colli G, et al. Conventional versus robot-assisted laparascopic Nissen fundoplication: a comparison of postoperative acid reflux parameters. Surg Endosc 2012;26:1675-81.

13. Markar SR, Karthikesalingam AP, Hagen ME, et al. Robotic versus laparoscopic Nissen fundoplication for gastroesophageal reflux disease: systematic review and meta-analysis. Int J Med Robot 2010;6:125-31.

14. Luketich JD, Pennathur A, Awais O, et al. Outcomes after minimally invasive esophagectomy: Review of over 1000 patients. Ann Surg 2012;256:95-103.

15. Yerokun BA, Sun Z, Jeffrey Yang CF, et al. Minimally invasive versus open esophagectomy for esophageal cancer; a population-based analysis. Ann Thorac Surg 2016;102:416-23.

16. Chao YK, Wen YW, Chuang WY, Cerfolio RJ. Transition from video-assisted thoracoscopic to robotic esophagectomy: a single surgeon's experience. Dis Esophagus 2020;33:doz033.

17. Okusanya OT, Sarkaria IS, Hess NR, et al. Robotic assisted minimally invasive esophagectomy (RAMIE): the University of Pittsburgh Medical Center initial experience. Ann Cardiothorac Surg 2017;6:179-85.

18. Wee JO, Bravo-Iniguez CE, Jaklitsch MT. Early experience of robot-assisted esophagectomy with circular end-end 
stapled anastomosis. Ann Thorac Surg 2016;102:253-9.

19. Jin D, Yao L, Yu J, et al. Robotic-assisted minimally invasive esophagectomy versus the conventional minimally invasive one: A meta-analysis and systematic review. Int J Med Robot 2019;15:e1988.

20. van der Sluis PC, van der Horst S, May AM, et al. Robotassisted minimally invasive thoracoscopic esophagectomy versus open transthoracic esophagectomy for resectable esophageal cancer A randomized controlled trial. Ann Surg 2019;269:621-30.

21. Oor JE, Wiezer MJ, Hazerbroek EJ. Hiatal hernia after open versus minimally invasive esophagectomy: a systematic review and meta-analysis. Ann Surg Oncol 2016;23:2690-8.
Cite this article as: Shemmeri E, Wee JO. Robotics and minimally invasive esophageal surgery. Ann Transl Med 2021;9(10):898. doi: 10.21037/atm-20-4138 